\title{
A new characterization of symplectic groups $\mathrm{C}_{2}\left(3^{\mathrm{n}}\right)$
}

\author{
Behnam Ebrahimzadeh and Reza Mohammadyari
}

\begin{abstract}
We prove that symplectic groups $C_{2}\left(3^{n}\right)$, where $n=2^{k}$ $(k \geq 0)$ and $\left(3^{2 n}+1\right) / 2$ is a prime number, can be uniquely determined by the order of the group and the number of elements with the same order.
\end{abstract}

\section{Introduction}

Let $G$ be a finite group, $\pi(G)$ be the set of prime divisors of the order of $G$ and $\pi_{e}(G)$ be the set of orders of elements in $G$. If $k \in \pi_{e}(G)$, then we denote the number of elements of order $k$ in $G$ by $m_{k}(G)$ and the set of the numbers of elements with the same order in $G$ by $n s e(G)$. In other words,

$$
n s e(G)=\left\{m_{k}(G): k \in \pi_{e}(G)\right\} .
$$

Also we denote a Sylow $p$-subgroup of $G$ by $G_{p}$ and the number of Sylow $p$-subgroups of $G$ by $n_{p}(G)$. The prime graph $\Gamma(G)$ of group $G$ is a graph whose vertex set is $\pi(G)$, and two vertices $u$ and $v$ are adjacent if and only if $u v \in \pi_{e}(G)$. Moreover, assume that $\Gamma(G)$ has $t(G)$ connected components $\pi_{i}$, for $i=1,2, \ldots, t(G)$. In the case where $G$ is of even order, we assume that $2 \in \pi_{1}$.

The characterization of groups by $n s e(G)$ pertains to Thompson's problem (see [6]) which Shi posed in [9]. The first time, this type of characterization was studied by Shao and Shi. In [8], they proved that if $S$ is a finite simple group with $|\pi(S)|=4$, then $S$ is characterizable by $n s e(S)$ and $|S|$. Following this result, in $[5,4,7]$ it is proved that sporadic simple groups, linear groups $L_{2}(p)$, where $2^{n}-1$ or $2^{n}+1$ is a prime number, and Suzuki groups $S z(q)$,

Received October 27, 2018.

2010 Mathematics Subject Classification. 20D06; 20 D60.

Key words and phrases. Order of an element; elements with the same order; prime graph; symplectic group.

http://dx.doi.org/10.12697/ACUTM.2019.23.12

Corresponding author: Behnam Ebrahimzadeh 
where $q-1$ is a prime number, can be uniquely determined by the order of the group and $n s e(G)$. In this paper, we prove that symplectic groups $C_{2}\left(3^{n}\right)$, where $n=2^{k}(k \geq 0)$ and $\left(3^{2 n}+1\right) / 2$ is a prime number can be uniquely determined by the order of the group and the number of elements with the same order. In fact, we prove the following theorem.

Main Theorem. Let $G$ be a group with $|G|=\left|C_{2}\left(3^{n}\right)\right|$ and nse $(G)=$ $n s e\left(C_{2}\left(3^{n}\right)\right)$, where $n=2^{k}(k \geq 0)$ and $p=\left(3^{2 n}+1\right) / 2$ is a prime number. Then $G$ is isomorphic to $C_{2}\left(3^{n}\right)$.

\section{Notation and preliminaries}

Lemma 2.1 (see [3]). Let $G$ be a Frobenius group of even order with kernel $K$ and complement $H$. Then

(a) $t(G)=2, \pi(H)$ and $\pi(K)$ are vertex sets of the connected components of $\Gamma(G)$;

(b) $|H|$ divides $|K|-1$;

(c) $K$ is nilpotent.

Definition 2.2. A group $G$ is called a 2-Frobenius group if there is a normal series $1 \unlhd H \unlhd K \unlhd G$ such that $G / H$ and $K$ are Frobenius groups with kernels $K / H$ and $H$, respectively.

Lemma 2.3 (see [1]). Let $G$ be a 2-Frobenius group of even order. Then (a) $t(G)=2, \pi(H) \cup \pi(G / K)=\pi_{1}$ and $\pi(K / H)=\pi_{2}$;

(b) $G / K$ and $K / H$ are cyclic groups satisfying $|G / K|$ divides $|A u t(K / H)|$.

Lemma 2.4 (see [10]). Let $G$ be a finite group with $t(G) \geq 2$. Then one of the following statements holds:

(a) $G$ is a Frobenius group;

(b) $G$ is a 2-Frobenius group;

(c) $G$ has a normal series $1 \unlhd H \unlhd K \unlhd G$ such that $H$ and $G / K$ are $\pi_{1}$-groups, $K / H$ is a non-abelian simple group, $H$ is a nilpotent group and $|G / K|$ divides $\mid$ Out $(K / H) \mid$.

Lemma 2.5 (see [2]). Let $G$ be a finite group and $m$ be a positive integer dividing $|G|$. If $L_{m}(G)=\left\{g \in G \mid g^{m}=1\right\}$, then $m|| L_{m}(G) \mid$.

Lemma 2.6. Let $G$ be a finite group. Then for every $i \in \pi_{e}(G), \varphi(i)$ divides $m_{i}(G)$, and $i$ divides $\sum_{j \mid i} m_{j}(G)$. Moreover, if $i>2$, then $m_{i}(G)$ is even.

Proof. By Lemma 2.5, the proof is straightforward. 
Lemma 2.7 (see [11]). Let $q, k, l$ be natural numbers. Then

(1) $\left(q^{k}-1, q^{l}-1\right)=q^{(k, l)}-1$.

(2) $\left(q^{k}+1, q^{l}+1\right)=\left\{\begin{array}{l}q^{(k, l)}+1 \text { if both } k /(k, l) \text { and } l /(k, l) \text { are odd, } \\ (2, q+1) \text { otherwise. }\end{array}\right.$

(3) $\left(q^{k}-1, q^{l}+1\right)=\left\{\begin{array}{l}q^{(k, l)}+1 \text { if } k /(k, l) \text { is even and } l /(k, l) \text { is odd, } \\ (2, q+1) \text { otherwise. }\end{array}\right.$

In particular, for every $q \geq 2$ and $k \geq 1$, the inequality $\left(q^{k}-1, q^{k}+1\right) \leq 2$ holds.

Lemma 2.8. Let $G$ be a symplectic group $C_{2}\left(3^{n}\right)$, where $p=\left(3^{2 n}+1\right) / 2$ is a prime number. Then $m_{p}(G)=(p-1)|G| /(8 p)$ and, for every $i \in$ $\pi_{e}(G)-\{1, p\}, p$ divides $m_{i}(G)$. Thus

Proof. Since $\left|G_{p}\right|=p$, we deduce that $G_{p}$ is a cyclic group of order $p$.

$$
m_{p}(G)=\varphi(p) n_{p}(G)=(p-1) n_{p}(G) .
$$

Now it is enough to show that $n_{p}(G)=|G| /(8 p)$. By [10], $p$ is an isolated vertex of $\Gamma(G)$. Hence $\left|C_{G}\left(G_{p}\right)\right|=p$ and $\left|N_{G}\left(G_{p}\right)\right|=x p$ for a natural number $x$. We know that $N_{G}\left(G_{p}\right) / C_{G}\left(G_{p}\right)$ embeds in $A u t\left(G_{p}\right)$, which implies $x \mid p-$ 1. Furthermore, by Sylow's theorem, $n_{p}(G)=\left|G: N_{G}\left(G_{p}\right)\right|$ and $n_{p}(G) \equiv 1$ $(\bmod p)$. Therefore $p$ divides $|G| /(x p)-1$. Thus $q^{2}+1 / 2$ divides $q^{4}\left(q^{4}-\right.$ 1) $\left(q^{2}-1\right) / 2 /(x p)-1$. It follows that $q^{2}+1$ divides $\left(2 q^{8}-4 q^{6}+2 q^{4}-x\right)$, hence $q^{2}+1$ divides $\left(q^{2}+1\right)\left(2 q^{6}-6 q^{4}+8 q^{2}-8\right)+(8-x)$, and since $x \mid p-1$, we obtain that $x=8$. Let $i \in \pi_{e}(G)-\{1, p\}$. Since $p$ is an isolated vertex of $\Gamma(G)$, we conclude that $p \nmid i$ and $p i \notin \pi_{e}(G)$. Thus $G_{p}$ acts fixed point freely on the set of elements of order $i$ by conjugation and hence $\left|G_{p}\right| \mid m_{i}(G)$. So we conclude that $p \mid m_{i}(G)$.

\section{Proof of the Main Theorem}

In this section, we prove the main theorem by the following lemmas. We denote by $C$ the symplectic group $C_{2}\left(3^{n}\right)$, where $n=2^{k}(k \geq 0)$ and $p:=$ $\left(3^{2 n}+1\right) / 2$ is a prime number. Recall that $G$ is a group with $|G|=|C|$ and $n s e(G)=n s e(C)$.

Lemma 3.1. We have

$$
m_{2}(G)=m_{2}(C), m_{p}(G)=m_{p}(C), n_{p}(G)=n_{p}(C),
$$

$p$ is an isolate vertex of $\Gamma(G)$, and $p \mid m_{k}(G)$ for every $k \in \pi_{e}(G)-\{1, p\}$.

Proof. By Lemma 2.6, for every $1 \neq r \in \pi_{e}(G), r=2$ if and only if $m_{r}(G)$ is odd. Thus we deduce that $m_{2}(G)=m_{2}(C)$. According to Lemma 2.6, $\left(m_{p}(G), p\right)=1$. Thus $p \nmid m_{p}(G)$ and hence Lemma 2.8 implies that $m_{p}(G) \in\left\{m_{1}(C), m_{2}(C), m_{p}(C)\right\}$. Moreover, $m_{p}(G)$ is even, so we conclude 
that $m_{p}(G)=m_{p}(C)$. Since $G_{p}$ and $C_{p}$ are cyclic groups of order $p$ and $m_{p}(G)=m_{p}(C)$, we deduce that $m_{p}(G)=\varphi(p) n_{p}(G)=\varphi(p) n_{p}(C)=$ $m_{p}(C)$, so $n_{p}(G)=n_{p}(C)$.

Now we prove that $p$ is an isolated vertex of $\Gamma(\mathrm{G})$. Assume the contrary. Then there is $t \in \pi(G)-\{p\}$ such that $t p \in \pi_{e}(G)$. So $m_{t p}(G)=$ $\varphi(t p) n_{p}(G) k$, where $k$ is the number of cyclic subgroups of order $t$ in $C_{G}\left(G_{p}\right)$ and since $n_{p}(G)=n_{p}(C)$, it follows that

$$
m_{t p}(G)=(t-1)(p-1)|C| k /(8 p) .
$$

If $m_{t p}(G)=m_{p}(C)$, then $t=2$ and $k=1$. Furthermore, Lemma 2.5 yields $p \mid m_{2}(G)+m_{2 p}(G)$ and since $m_{2}(G)=m_{2}(C)$ and $p \mid m_{2}(C)$, we have $p \mid m_{2 p}(G)$, which is a contradiction. So Lemma 2.8 implies that $p \mid m_{t p}(G)$. Hence $p \mid t-1$, and since $m_{t p}(G)<|G|$, we have that $t-1 \leq 8$. In conclusion we deduce that $t \in\{3,4,5,6,7,8,9\}$. Now, since $p \nmid m_{t p}(G)$, this is a contradiction.

Let $k \in \pi_{e}(G)-\{1, p\}$. Since $p$ is an isolated vertex of $\Gamma(G)$, we have that $p \nmid k$ and $p k \notin \pi_{e}(G)$. Thus $G_{p}$ acts fixed point freely on the set of elements of order $k$ by conjugation and hence $\left|G_{p}\right| \mid m_{k}(G)$. So we conclude that $p \mid m_{k}(G)$.

Lemma 3.2. The group $G$ is neither a Frobenius group nor a 2-Frobenius group.

Proof. Let $G$ be a Frobenius group with kernel $K$ and complement $H$. Then by Lemma 2.1, $t(G)=2$ and $\pi(H)$ and $\pi(K)$ are vertex sets of the connected components of $\Gamma(G)$, and $|H|$ divides $|K|-1$. Now by Lemma $3.1, p$ is an isolated vertex of $\Gamma(G)$. Thus we deduce that (i) $|H|=p$ and $|K|=|G| / p$, or (ii) $|H|=|G| / p$ and $|K|=p$. Since $|H|$ divides $|K|-1$, we conclude that the last case can not occur. So $|H|=p$ and $|K|=|G| / p$, hence

$$
\left(q^{2}+1\right) / 2 \mid \frac{q^{4}\left(q^{4}-1\right)\left(q^{2}-1\right) / 2}{\left(q^{2}+1\right) / 2}-1 .
$$

We conclude that

$$
\left(q^{2}+1\right) \mid\left(\left(q^{2}+1\right)\left(2 q^{6}-6 q^{4}+8 q^{2}-8\right)+7 .\right.
$$

Thus $q^{2}+1 \mid 7$ which is impossible.

We now show that $G$ is not a 2-Frobenius group. Let $G$ be a 2-Frobenius group. Then $G$ has a normal series $1 \unlhd H \unlhd K \unlhd G$ such that $G / H$ and $K$ are Frobenius groups by kernels $K / H$ and $H$, respectively. Set $|G / K|=x$. Since $p$ is an isolated vertex of $\Gamma(G)$, we have $|K / H|=p$ and $|H|=|G| /(x p)$. By Lemma 2.3, $|G / K|$ divides $|A u t(K / H)|$. Thus $x \mid p-1$ and since, by Lemma 2.7, $(p-1, q-1)=1$, we have $\left(q^{2}-1 / 2, q^{2}+1 / 2\right)=1$. Now, since $|G / K| \mid(p-1)$, we deduce that $q^{2}+1 / 2 \mid H$. The group $H$ is nilpotent. Therefore, $H_{t} \rtimes K / H$ is a Frobenius group with kernel $H_{t}$ and complement 
$K / H$, where $t=q^{2}+1 / 2$. So $|K / H|$ divides $\left|H_{t}\right|-1$. It implies that $q^{2}+1 / 2 \leq\left(q^{2}+1\right) / 2-1$, but this is a contradiction.

Lemma 3.3. The group $G$ is isomorphic to the group $C$.

Proof. By Lemma 3.1, $p$ is an isolated vertex of $\Gamma(G)$. Thus $t(G)>1$ and $G$ satisfies one of the cases of Lemma 2.4. Now, Lemma 3.2 implies that $G$ is neither a Frobenius group nor a 2-Frobenius group. Thus only the case (c) of Lemma 2.4 occurs. So $G$ has a normal series $1 \unlhd H \unlhd K \unlhd G$ such that $H$ and $G / K$ are $\pi_{1}$-groups, and $K / H$ is a non-abelian simple group. Since $p$ is an isolated vertex of $\Gamma(G)$, we have $p|| K / H \mid$. According to the classification of the finite simple groups we know that the possibilities are: alternating groups $A_{n}$, where $n>5 ; 26$ sporadic finite simple groups; simple groups of Lie type. We deal with the above cases separately.

Step 1. Let $K / H \cong A_{n}$, where $n \geq 5, n=p^{\prime}, p^{\prime}+1, p^{\prime}+2$. For this purpose, we consider $\left(q^{2}+1\right) / 2=p^{\prime}$. Then we deduce $p^{\prime}+1=\left(q^{2}+3\right) / 2$. Now $p^{\prime}+1|| A_{n}|||G|$, but we can see easily that $\frac{q^{2}+3}{2} \nmid|G|$, which is a contradiction. Now we consider $\left(q^{2}+1\right) / 2=p^{\prime}-2$, so $p^{\prime}=\left(q^{2}+4\right) / 2$. Since $p^{\prime}|| A_{n}|| G \mid$, but we can see easily that $\frac{q^{2}+4}{2} \nmid|G|$, we obtain a contradiction.

Step 2. If $K / H$ is a sporadic group, then we consider $\left(q^{2}+1\right) / 2=$ $5,7,11,13,17,19,23,29,31,37,41,43,47,59,71$, where this number is the order of components of sporadic groups. If, for example, $\left(q^{2}+1\right) / 2=5$, then we deduce $q^{2}=9$, and hence $q=3$. Now, since $M_{11} \nmid|G|$, this is a contradiction. For the other groups we have a contradiction, similarily.

Step 3. Here we suppose that $K / H$ is isomorphic to a group of Lie type. For this purpose, we consider the following cases.

Case 1. Let $K / H \cong B_{n}\left(q^{\prime}\right)$, where $n \geq 2$, or $C_{n}\left(q^{\prime}\right)$, where $n \geq 3$. If $K / H \cong B_{n}\left(q^{\prime}\right)$, where $n \geq 2$, then we consider $\left(q^{2}+1\right) / 2=\left(q^{\prime n}+1\right) / 2$ so $q^{2}=q^{\prime n}$. On the other hand,

$$
\left|B_{n}\left(q^{\prime}\right)\right|=\frac{1}{\left(2, q^{\prime}-1\right)} q^{\prime n^{2}} \prod_{i=1}^{n}\left(q^{\prime 2 i}-1\right) \mid q^{4}\left(q^{4}-1\right)\left(q^{2}-1\right)
$$

and also we know that each $p$-part of $K / H$ divides $p$-part of $G$. Since $p=\left(q^{2}+1\right) / 2$, we have $q^{2}=2 p-1$. Now, since $\left|B_{n}\left(q^{\prime}\right)\right||| G \mid$, we conclude that

$$
4 p(p-1)(p-2)(2 p-1)^{2}=q^{\prime n^{2}} \prod_{i=1}^{n}\left(q^{\prime 2 i}-1\right) .
$$

Thus we have $p \mid q^{\prime n^{2}}$ or $p \mid \prod_{i=1}^{n}\left(q^{\prime 2 i}-1\right)$. On the other hand, $q^{\prime n^{2}} \mid p-1$ or $p-2$ or $(2 p-1)$. If $p \mid q^{\prime n^{2}}$, then $p \nmid q^{\prime}$, and so $p \mid \prod_{i=1}^{n}\left(q^{\prime 2 i}-1\right)$. In other words, $p \mid q^{\prime 2 t}-1$, where $1 \leq t \leq n$. From $q^{\prime n^{2}} \mid p-1$ it follows that

$$
q^{\prime n^{2}} \leq p-1 \leq p \leq q^{\prime 2 t}-1 \leq q^{\prime 2 n}-1 \leq q^{\prime 2 n} .
$$


As a result $q^{\prime n^{2}} \leq q^{\prime 2 n}$, so $n^{2} \leq 2 n, n \leq 2$, but this is a contradiction. Similarily, there is a contradiction for other cases.

Case 2. Let $K / H \cong D_{n}\left(q^{\prime}\right)$, where $n \geq 4$ or ${ }^{2} D_{n}\left(q^{\prime}\right)$ with $n \geq 4$. Then we consider $\left(q^{2}+1\right) / 2=\left(q^{\prime n}-1\right) /(q-1)$. On the other hand,

$$
\left|D_{n}\left(q^{\prime}\right)\right|=\frac{1}{\left(4, q^{\prime n}-1\right)} q^{\prime n(n-1)}\left(q^{\prime n}-1\right) \prod_{i=1}^{n-1}\left(q^{\prime 2 i}-1\right) \mid q^{4}\left(q^{4}-1\right)\left(q^{2}-1\right)
$$

and also we know that each $p$-part of $K / H$ divides $p$-part of $G$. Since $\left|D_{n}\left(q^{\prime}\right)\right||| G \mid$, it follows that

$$
4 p(p-1)(p-2)(2 p-1)^{2}=\frac{1}{\left(4, q^{\prime n}-1\right)} q^{\prime n(n-1)}\left(q^{\prime n}-1\right) \prod_{i=1}^{n-1}\left(q^{\prime 2 i}-1\right) .
$$

Now we have

$$
p \mid q^{\prime n(n-1)} \text { or } p \mid q^{\prime n}-1 \text { or } p \mid \prod_{i=1}^{n-1}\left(q^{\prime 2 i}-1\right) .
$$

On the other hand, $q^{\prime n(n-1)} \mid p-1$ or $p-2$ or $(2 p-1)$. If $p \mid q^{\prime n(n-1)}$, then $p \nmid q^{\prime}$, so $p \mid \prod_{i=1}^{n-1}\left(q^{\prime 2 i}-1\right)$. In other words, $p \mid q^{\prime 2 t}-1$, where $1 \leq t \leq n-1$. Since $q^{\prime n(n-1)} \mid p-1$, we have

$$
q^{\prime n(n-1)} \leq p-1 \leq p \leq q^{\prime 2 t}-1 \leq q^{\prime 2 n}-1 \leq q^{\prime 2 n} .
$$

As a result $q^{\prime n(n-1)} \leq q^{\prime 2 n}$, so $n(n-1) \leq 2 n, n \leq 3$, but this is a contradiction $(n \geq 4)$. There is a contradiction for other cases. Similarily, $K / H \varlimsup^{2} D_{n}(q)$.

Case 3. Let $K / H \cong^{2} A_{n}\left(q^{\prime}\right)$, where $n \geq 2$. Then we consider

$$
\frac{q^{2}+1}{2}=\frac{q^{\prime n+1}+1}{\left(q^{\prime}+1\right)(q+1, n+1)}
$$

and so

$$
q^{2}+1=\frac{q^{\prime n+1}+1}{\left(q^{\prime}+1\right)(q+1, n+1)}<q^{\prime n+1}+1, \quad q^{2}<q^{\prime n+1} .
$$

Since $n \geq 2$, we get

$$
q^{\prime n(n+1) / 2}>q^{\prime 4 n / 2} \geq q^{\prime 2 n}>q^{\prime n}>2^{4 n} .
$$

But, on the other hand, we have

$$
q^{\prime n(n+1) / 2}=|K / H|_{r} \leq|G|_{r} \leq 2^{3 n},
$$

which is a contradiction.

Case 4. Let $K / H \cong E_{6}(q), E_{7}(q), E_{8}(q)$, where $n \geq 2$, or let $K / H \cong$ $F_{4}(q)$. If $K / H \cong E_{8}\left(q^{\prime}\right)$, then we consider $\left(q^{2}+1\right) / 2=q^{\prime 8}-q^{\prime 4}+1$. On the other hand,

$$
\left|E_{8}\left(q^{\prime}\right)\right|=q^{\prime 120}\left(q^{\prime 30}-1\right)\left(q^{\prime 24}-1\right)\left(q^{\prime 20}-1\right)\left(q^{\prime 18}-1\right)\left(q^{\prime 14}-1\right)
$$




$$
\times\left(q^{\prime 12}-1\right)\left(q^{\prime 8}-1\right)\left(q^{\prime 2}-1\right) \mid q^{4}\left(q^{4}-1\right)\left(q^{2}-1\right) .
$$

Since $\left|E_{8}\left(q^{\prime}\right)\right| \nmid|G|$, this is a contradiction. For other cases we have similarily a contradiction.

Case 5. Let $K / H \cong G_{2}\left(q^{\prime}\right)$, where $q^{\prime} \equiv \pm 2(\bmod 5)$. Then we consider $\left(q^{2}+1\right) / 2=q^{\prime}\left(q^{\prime} \mp 1\right)$. Since $\left(q^{\prime}, q^{\prime} \mp 1\right)=1$, we obtain $q^{2}-1=2 q^{\prime}\left(q^{\prime} \pm 1\right)$, Now $\left|G_{2}\left(q^{\prime}\right)\right| \nmid|G|$, which is a contradiction.

Case 6 . Let $K / H \cong{ }^{2} B_{2}\left(q^{\prime}\right)$, where $q^{\prime}=2^{2 r+1}, r \geq 1$. Then we consider $\left(q^{2}+1\right) / 2=q^{\prime} \pm \sqrt{2 q^{\prime}}+1$. As a result $q^{2}-1=2^{m+1}\left(2^{m} \pm 1\right)$. Since $\left(2^{m+1}, 2^{m} \pm 1\right)=1$, we deduce that

$$
(q-1)(q+1)=2^{m+1}\left(2^{m} \pm 1\right) .
$$

In other words,

$$
\left(3^{n}-1\right)\left(3^{n}+1\right)=2^{m+1}\left(2^{m} \pm 1\right) .
$$

Consequently, $3^{n}-1=2^{m} \pm 1$ and $3^{n}+1=2^{m+1}$, which is a contradiction.

Case 7. Let $K / H \cong 2 F_{4}\left(q^{\prime}\right)$, where $q^{\prime}=2^{2 s+1}, s \geq 1$. Then we consider

$$
\frac{q^{2}+1}{2}=q^{\prime 2} \pm \sqrt{2 q^{\prime 3}}+q^{\prime} \pm \sqrt{2 q^{\prime}}+1 .
$$

As a result $q^{2}-1=q^{\prime 2} \pm \sqrt{2 q^{\prime 3}}+q^{\prime} \pm \sqrt{2 q^{\prime}}$, so we deduce

$$
(q-1)(q+1)=2^{s+1}\left(2^{3 s+1} \pm 2^{2 s+1}+2^{s}+1\right) .
$$

It follows that $3^{n}-1=2^{s+1}$ and $3^{n}+1=\left(2^{3 s+1} \pm 2^{2 s+1}+2^{s}+1\right)$, where we can see easily a contradiction.

Case 8 . Let $K / H \cong 2 E_{6}(q)$, where $q=2^{2 t+1}, t \geq 1$. Then we consider

$$
\frac{q^{2}+1}{2}=\frac{q^{6}-q^{\prime 3}+1}{\left(3, q^{\prime}+1\right)}
$$

so $q^{2}<q^{\prime 6}-q^{\prime 3}+1<q^{\prime 6}$. Hence $q^{\prime 36}>3^{12 n}$.

On the other hand,

$$
\begin{aligned}
\left|{ }^{2} E_{6}\left(q^{\prime}\right)\right|= & \frac{1}{\left(3, q^{\prime}+1\right)} q^{\prime 36}\left(q^{12}-1\right)\left(q^{\prime 9}+1\right) \\
& \times\left(q^{\prime 8}-1\right)\left(q^{\prime 6}-1\right)\left(q^{\prime 5}+1\right)\left(q^{\prime 2}-1\right) .
\end{aligned}
$$

Now, we obtain $q=r^{s}$. Therefore, by Lemma 2.4,

$$
q^{36}=r^{36 s}=|K / H|_{r} \leq|G|_{r} \leq 2^{3 m},
$$

which is a contradiction.

Case 9. Let $K / H \cong 3 D_{4}\left(q^{\prime}\right)$. Then we consider $\left(q^{2}+1\right) / 2=q^{4}-q^{\prime 2}+1$, as a result

$$
\left(3^{n}-1\right)\left(3^{n}+1\right)=2 q^{2}\left(q^{\prime 2}-1\right) .
$$

Hence $2 q^{\prime 2}=3^{n}+1$ and $q^{\prime 2}-1=3^{n}-1$, which is a contradiction. 
Case 10. Let $K / H \cong L_{n+1}\left(q^{\prime}\right)$. Then we consider

$$
\frac{q^{2}+1}{2}=\frac{q^{\prime n+1}-1}{\left(q^{\prime}-1\right)\left(q^{\prime}-1, n+1\right)}
$$

So

$$
q^{\prime n+1}-1>\frac{q^{\prime n+1}-1}{(q-1)(q-1, n+1)}=q^{2}+1 .
$$

As a result $q^{2}<q^{\prime n+1}$, so

$$
q^{\prime n(n+1) / 2}>q^{4(n+1) / 2}>q^{2 n}>3^{8 n} .
$$

On the other hand, by Lemma 2.4 we have

$$
q^{n(n+1) / 2}=|K / H|_{r} \leq|G|_{r} \leq 2^{3 n}
$$

which is a contradiction.

Hence $K / H \cong C_{2}\left(3^{m}\right)$, in conclusion $|K / H|=\left|C_{2}\left(3^{m}\right)\right|$. We know that $H \unlhd K \unlhd G$. Since $p$ is an isolated vertex of $\Gamma(G)$, we deduce that $p|| K / H \mid$. Hence $\left(q^{2}+1\right) / 2=\left(q^{\prime 2}+1\right) / 2$. As a result $q=q^{\prime}$, so $n=m$. Now, since $|K / H|=|C|$ and $1 \unlhd H \unlhd K \unlhd G$, we conclude that $H=1$ and $G=K \cong C$.

\section{References}

[1] G. Y. Chen, About Frobenius groups and 2-Frobenius groups, J. Southwest China Normal Univ. 20(5) (1995), 485-487.

[2] G. Frobenius, Verallgemeinerung des Sylow'schen Satzes, Berliner Sitzungsberichte (1895), 981-983.

[3] D. Gorenstein, Finite Groups, Chelsea Publishing Co., New York, 1980.

[4] A. Khalili Asboei, S. S. Amiri, A. Iranmanesh and A. Tehranian, A new characterization of sporadic simple groups by nse and order, J. Algebra Appl. 12(2) (2013), 1250158,3 pp.

[5] A. Khalili Asboei and A. Iranmanesh, Characterization of the linear groups $L_{2}(p)$, Czechoslovak Math. J. 64(139) (2014), 459-464.

[6] V. D. Mazurov and E. I. Khukhro, Unsolved problems in group theory, The Kourovka Notebook, 16 ed. Inst. Mat. Sibirsk. Otdel. Akad. Novosibirsk, 2006.

[7] H. Parvizi Mosaed, A. Iranmanesh, and A. Tehranian, Characterization of Suzuki group by nse and order of group, Bull. Korean Math. Soc. 53(3) (2016), 651-656.

[8] C. G. Shao, W. J. Shi, and Q. H. Jiang, Characterization of simple $K_{4}$-groups, Front. Math. China 3(3) (2008), 355-370.

[9] W. J. Shi, A new characterization of the sporadic simple groups, in: Group Theory (Singapore, 1987), Walter de Gruyter, Berlin, 1989, pp. 531-540.

[10] J. S. Williams, Prime graph components of finite groups, J. Algebra 69(2) (1981), 487-513.

[11] A. V. Zavarnitsine, Recognition of the simple groups $L_{3}(q)$ by element orders, J. Group Theory $7(1)$ (2004), 81-97.

Department of Mathematics, Buinzahra Branch, Islamic Azad University, BUINZAHRA, IRAN

E-mail address: behnam.ebrahimzadeh@gmail.com

E-mail address: rezamohammadyari@gmail.com 